\title{
Low energy observables with the ATLAS experiment
}

\author{
Andrew Foster on behalf of the ATLAS Collaboration \\ University of Birmingham, School of Physics and Astronomy, Birmingham, B15 2TT, UK
}

\begin{abstract}
Recent results in the soft QCD sector of the Standard Model are presented. Measurements of low energy observables were performed with the ATLAS detector at the Large Hadron Collider at CERN. In particular, underlying event, charged particle correlation and diffraction observables are reported and various models of Monte Carlo predictions are compared to the data.
\end{abstract}

Keywords: Soft QCD, underlying event, Bose-Einstein correlations, AFP, diffraction

\section{Introduction}

Soft QCD processes, those with low four momentum transfer, can be characterised by low energy observables with which non-perturbative QCD can be probed. This regime cannot be modelled using perturbative QCD due to the large value of the strong coupling constant in soft processes and instead is typically described using phenomenological models, which are heavily steered by experimental inputs. These models are implemented in Monte Carlo (MC) generators which may be tuned to provide different descriptions of the data, enabling the advancement of the phenomenological models.

Due to the wide variety of interactions and different fractions of the proton momentum carried by colliding partons, the LHC enables the study of the transition between the hard scattering perturbative QCD region with large four momentum transfer and the aforementioned soft QCD region. Many previous measurements by ATLAS [1] have been performed in this field with some highlights being those of the underlying event [2], inelastic and total cross section measurements [3, 4], the inclusive charged particle distribution in minimum bias events [5] and diffraction [6]. Here the latest results on the underlying event [7], charged particle correlations $[8,9]$ and the prospects for future diffraction measurements [10] are presented.

The data were collected by the ATLAS experiment in proton-proton collisions at $\sqrt{\mathrm{s}}=0.9,7,8 \& 13 \mathrm{TeV}$. Many of these analyses use minimum bias events, which in ATLAS are defined as events selected by Minimum Bias Trigger Scintillators (MBTS); these are scintillator planes located outside of the inner tracking detector's endcaps.

\section{Underlying Event}

The underlying event (UE) is defined as the collision products that accompany a hard scattering process such as initial/final state radiation (I/FSR), multi parton interactions (MPI) and spectator remnants from the interacting proton. A good understanding of the UE distributions, particularly MPI, is important in its own right as well as its role as a background for other physics final states at the LHC.

As with previous UE analyses by ATLAS [2] and other experiments $[11,12]$, the outgoing particles are separated into different regions in $\phi$ relative to the leading charged particle characterised by the variable $\Delta \phi$, see Figure 1. This separation between towards, away and transverse typically results in more activity in the towards and away region from the primary charged particle due to jet activity and conservation of momentum, and then less activity in the transverse regions making them more representative of the UE. The MBTS trigger is used to select the sample. 


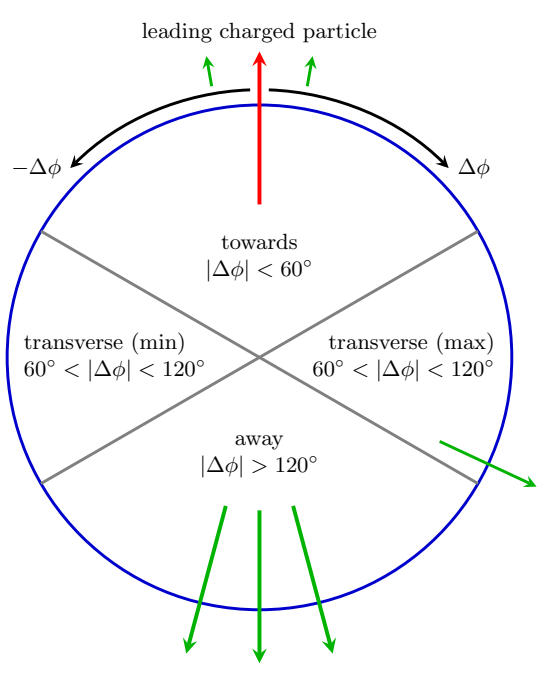

Figure 1: Regions of separation in azimuthal angle, defined from the primary charged particle [7].

The two main observables considered in this analysis are the number of charged particles, $N_{\mathrm{ch}} / \delta \eta \delta \phi$, and the sum of the particles' transverse momenta, $\Sigma p_{\mathrm{T}} / \delta \eta \delta \phi$, both calculated per unit area of azimuthal angle, $\phi$, and pseudorapidity, $\eta$. As a function of the leading particle's $p_{\mathrm{T}}, p_{\mathrm{T}}^{\text {lead }}$, a plateau is observed in the transverse region above a few $\mathrm{GeV}$, Figure 2, as the UE is relatively independent of $p_{\mathrm{T}}^{\text {lead }}$. A similar effect is observed for the $\Sigma p_{\mathrm{T}}$ observable.

Of the two transverse regions, the side with less activity is defined as 'trans-min', and this is the most sensitive to the underlying event, as it contains less contamination from the leading hard scattering event. This analysis also introduces a conceptual 'trans-diff' region defined as the difference between the two transverse regions. This enables the measurement of the degree of contamination of the transverse region due to the primary hard scattering process, as the UE should on average be the same in both transverse regions.

Figure 3 displays the charged particle density in this trans-diff region as a function of $p_{\mathrm{T}}^{\text {lead }}$, and it can be seen that the number of charged particles increases with $p_{\mathrm{T}}^{\text {lead }}$, implying that the difference between the two transverse regions does reflect the contamination due to the leading hard process.

Throughout the analysis, it is observed that the MC tunes that largely perform the best in different regions are the Pythia 8 [13], Monash and A14, tunes. However, none of the tunes model the trans-diff region well.

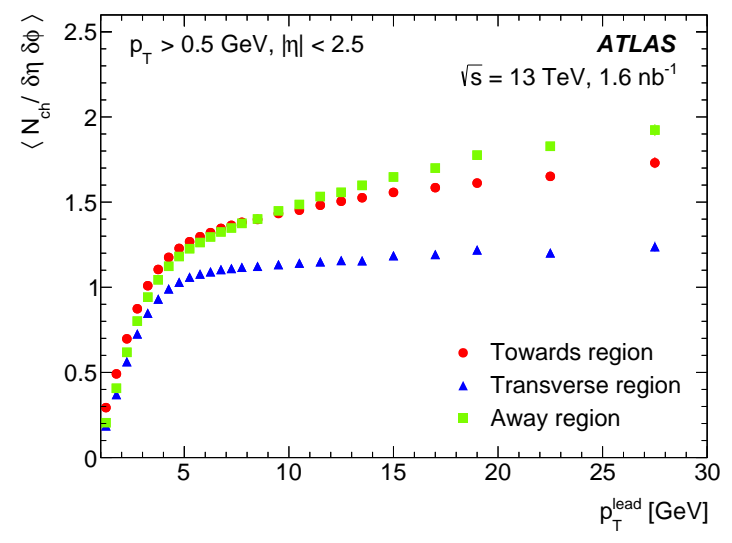

Figure 2: Number of charged particles per unit area of $\eta$ and $\phi$ for the towards, away and transverse regions [7].

\section{Correlation Studies}

\subsection{Bose-Einstein Correlations}

Bose-Einstein correlation (BEC) is an effect observed as the enhancement of the production of identical bosons, close in momentum space, from a chaotic source [14], resulting from correlations between the two bosons wave functions. This analysis considers pairs of same-sign charged pions relative to pairs of oppositesign charged pions, which should be almost entirely unaffected by BEC [15].

The effects of BEC can be formalised as a two particle correlation function of $Q$, the four-momentum difference squared between the two pions,

$$
C_{2}(Q)=\frac{\rho(Q)}{\rho_{0}(Q)}=C_{0}[1+\Omega(\lambda, Q R)](1+\epsilon Q),
$$

where $\rho(Q)$ and $\rho_{0}(Q)$ are the two-particle density functions for like-sign charged pions with BEC effects and opposite-sign charged pions respectively. $C_{0}$ is a normalisation constant, $\Omega$ is a Fourier transform of the space-time points of the emission source, where $R$ is an effective radius parameter and $\lambda$ is a strength parameter and $\epsilon$ accounts for long-distance correlations. The Goldhaber (radial Gaussian) and exponential parameterisation of $\Omega(\lambda, Q R)$ are considered in comparison to data.

The double ratio constant,

$$
R_{2}(Q)=\frac{C_{2}(Q)}{C_{2}^{M C}(Q)}=\frac{\rho(++,--) \rho^{M C}(+-)}{\rho(+-) \rho^{M C}(++,--)},
$$

is defined to account for the effects of resonances and isolate enhancements in the rate of like-sign charged 


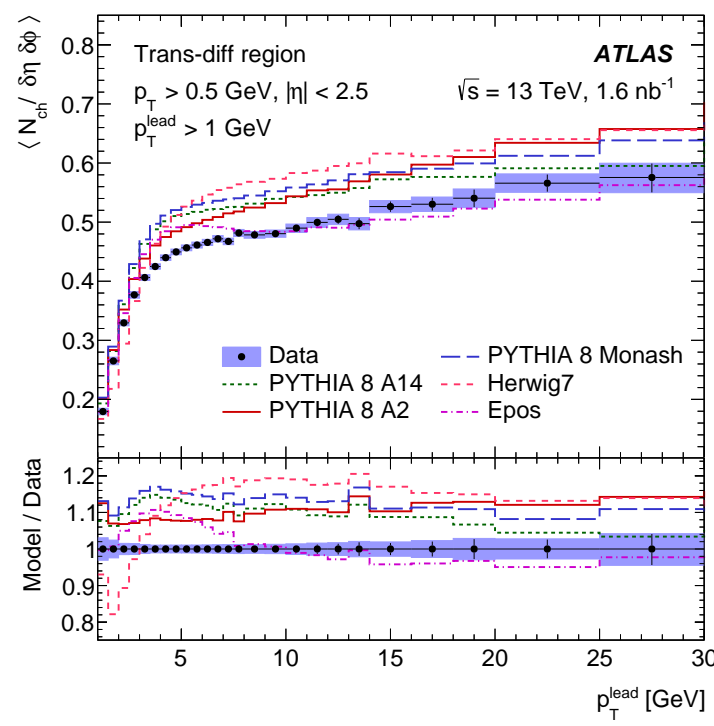

Figure 3: Number of charged particles per unit area of $\eta$ and $\phi$ in the trans-diff region [7].

pion emission. This is possible as the MC simulations used do not contain BEC effects and thus when compared to data should result in unity if no BEC are present.

The MBTS trigger is used in this analysis, and events are required to have at least one vertex with two tracks having $p_{\mathrm{T}}>100 \mathrm{MeV}$ in the ATLAS tracking detector. No particle identification is performed and thus all charged particles are treated as pions.

Figure 4 displays the expected enrichment of samesign pions in the low $\mathrm{Q}$ region. It can be seen that the data strongly favour the exponential parameterisation of $\Omega(\lambda, Q R)$ and thus this parameterisation model is used for extracting the parameters, $\lambda$ and $R$, from this function. The visible bump around $\mathrm{Q} \sim 0.7 \mathrm{GeV}$ is excluded from the fit due to an overestimation of $\rho \rightarrow \pi^{+} \pi^{-}$decays in the MC simulation.

The fit applied to Figure 4 is also applied to samples at varying multiplicities and also at $\sqrt{s}=0.9 \mathrm{TeV}$ and the $R$ and $\lambda$ parameters are extracted, see Figures 5 and 6 . The $\lambda$ parameter is observed to decrease with multiplicity. The $R$ parameter increases independently of the centre of mass energy up to multiplicities of $\sim 50$. Beyond this point, the radius appears to saturate, which has not been observed before. This saturation is predicted by some Pomeron-based models [16, 17].

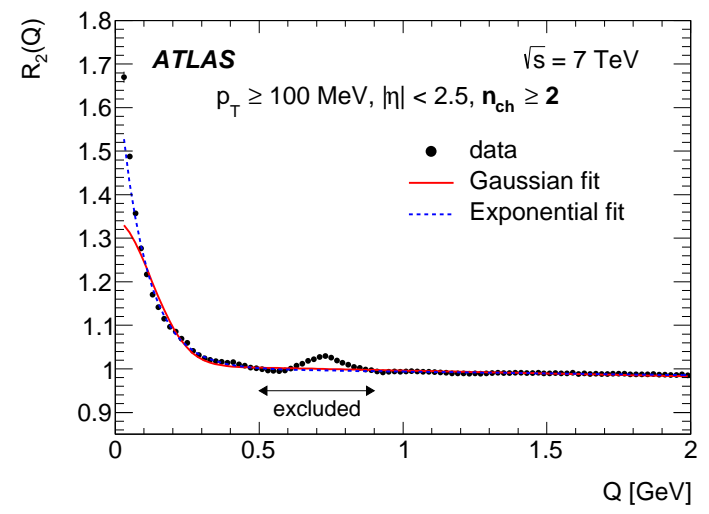

Figure 4: Measured double ratio constant, $R_{2}(Q)$, fitted with Gaussian and exponential parameterisations of $\Omega(\lambda, Q R)$, for events with multiplicity $\geq 2$ tracks at $\sqrt{s}=7 \mathrm{TeV}$ [8]

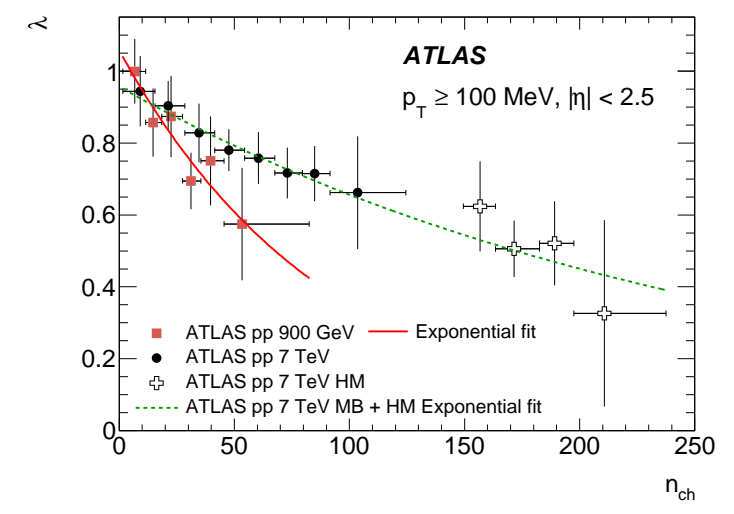

Figure 5: Multiplicity dependence of the strength parameter, $\lambda$, obtained from the exponential fit to $R_{2}(Q)$ [8].

\subsection{Ordered Hadron Chains}

The BEC effect discussed in Section 3.1 has never been well reproduced by MC generators, despite the effect being known and measured for many years. This implies that something is lacking in the MC modelling of the fragmentation and hadronisation process, from which hadrons are formed.

There are several models which attempt to nonarbitrarily reproduce the correlations between samesign hadrons, one of which is the quantised fragmentation of a three dimensional helical QCD string [18].

This analysis compares predictions from the $3 \mathrm{D}$ helical string model with the Lund String Model used in PyтHIA and the cluster hadronisation model, represented by Herwig++ [19] at $\sqrt{s}=7 \mathrm{TeV}$. The sample is the same as in Section 3.1 but with the additional require- 


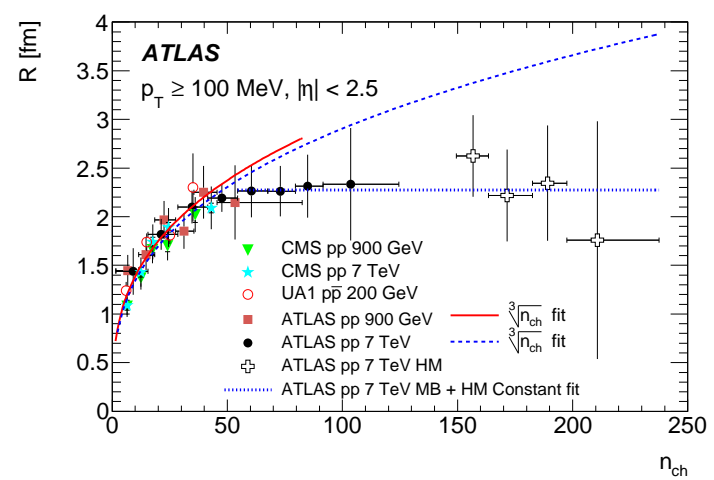

Figure 6: Multiplicity dependence of the effective radius parameter, $\mathrm{R}$, obtained from the exponential fit to $R_{2}(Q)$ [8].

ment that there be no second primary vertex with more than three tracks.

The 3D string model provides predictions for the four-momentum separation, $\mathrm{Q}$, between adjacent hadrons that are formed contiguously on the string during the fragmentation process. It is predicted that there is a quantum threshold in four-momentum difference for the production of opposite-sign adjacent hadrons. It is also predicted that chains of hadrons are emitted as three adjacent ground-state pions. These are selected in this analysis by minimising the four-momentum difference between all pairs of like-signed pions in the final state and then minimising the three-body mass when selecting a third, oppositely charged pion to form a chain of three pions. The model also predicts the maximum mass of these three-hadron chains to be $M_{3 h}<575 \pm 20 \mathrm{MeV}$.

Figure 7 displays the ratio, $R$ :

$$
R=\frac{N(Q)^{++,--}}{N(Q)^{+-}}
$$

between like and opposite signed hadron pairs as a function of Q for all data (black) and for data with hadron chains subtracted, where the chains are defined differently for the solid and hollow red data points. It can be seen that the only region affected by these chains is the region $\mathrm{Q}<0.3 \mathrm{GeV}$, which is also where the $\mathrm{BEC}$ enrichment is observed in Figure 4 from Section 3.1.

The results from this analysis indicate that the enhancement in the low-Q region typically attributed to BEC may also be understood in the formalism of a 3D QCD string producing chains of three hadrons via quantised fragmentation.

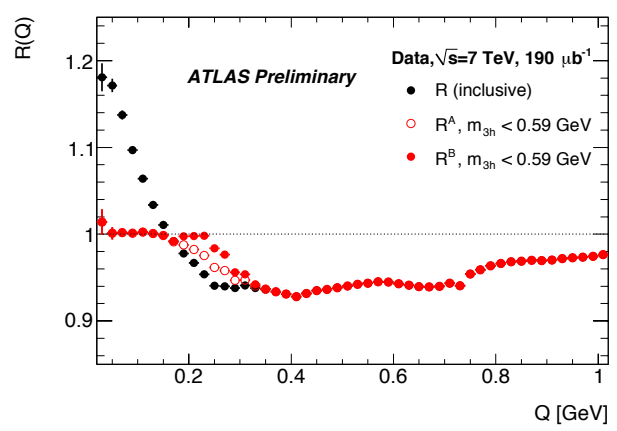

Figure 7: The ratio of like and opposite signed charged hadrons before and after the subtraction of chains with a mass below $0.59 \mathrm{GeV} . R^{A}$ and $R^{B}$ represent different constructions of the chains [9].

\section{Diffraction Prospects with AFP}

The ATLAS Forward Proton (AFP) detectors, installed during LHC shutdowns between 2015-2017, consist of a high resolution pixel silicon tracker combined with a Time-Of-Flight system located close to the outgoing proton beam, $210 \mathrm{~m}$ from the ATLAS interaction point in both directions [20]. The detector purpose is to identify diffractively scattered, intact protons that have been slightly perturbed from the main LHC proton beam. The analysis presented here is a proof-ofprinciple that AFP can identify diffractively scattered protons and was performed during a dedicated low pileup run with only one side of the AFP trackers installed.

One of the main observables that is used to differentiate diffractive scattering events from non-diffractive interactions is, $\xi$, the fractional energy loss of the proton relative to the beam energy. Detectors such as AFP and ALFA [21] at ATLAS, and TOTEM [22] at CMS, enable the tagging of protons and can calculate $\xi$ directly from the proton. At the time of this analysis, the AFP detector had not yet been aligned and so no proton energy information was available. This analysis utilises the relationship between $\xi$ and the calorimeter information from the system in the central ATLAS detector which is used in previous analyses [23] and is given by the expression,

$$
\xi_{\mathrm{cal}}=\frac{1}{\sqrt{s}} \sum_{i} p_{\mathrm{T}}^{i} e^{-\eta i},
$$

where all calorimeter clusters with $p_{\mathrm{T}}>200 \mathrm{MeV}$ are summed. The purpose of this analysis is to demonstrate that AFP is capable of selecting a diffraction enriched sample. This is investigated by comparing a sample triggered by the AFP detectors and a minimum bias sample triggered by the MBTS. Both samples are required to 
have a jet with transverse momentum above $10 \mathrm{GeV}$ and the AFP-triggered sample is required to pass a tighter offline AFP selection.

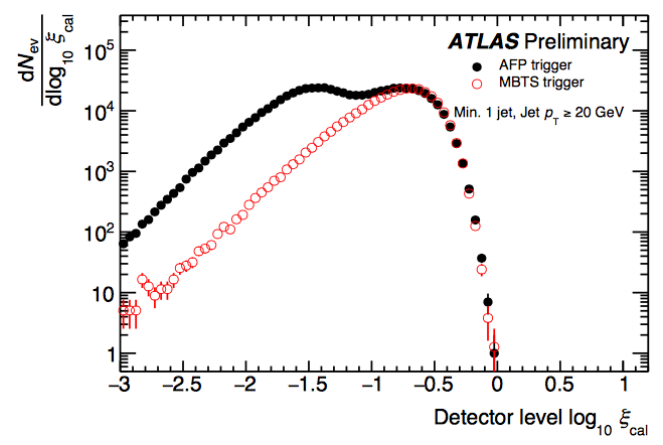

Figure 8: $\xi_{\text {cal }}$ in AFP and minimum bias triggered samples. The MB data is normalised to the AFP data in the large $\xi_{\text {cal }}$ region [9].

Figure 8 displays $\xi_{\text {cal }}$ for both samples. The AFPtriggered sample exhibits a clear double peak structure. This is indicative of selection of both diffractive events, at low $\xi_{\text {cal }}$, and minimum bias events, at high $\xi_{\text {cal }}$, which are detected due to background pile-up between minimum bias interactions and AFP triggers. The MBTStriggered sample only displays the minimum bias peak, and this is normalised to the AFP sample. This double peak structure in the AFP sample demonstrates that AFP is capable of selecting diffractive events and opens the door to future diffractive measurements. When run with the Time-Of-Flight system, the AFP detectors will be able to operate in nominal pile-up runs and distinguish between vertices along the $z$-axis.

\section{Conclusions}

A selection of the latest analyses from the ATLAS experiment in the field of soft-QCD, varying over a wide range of centre of mass energies, have been presented.

The underlying event has been measured and compared to MC predictions at $\sqrt{s}=13 \mathrm{TeV}$ and it is observed that the new 'trans-diff region' is a powerful tool to understand deficits in current MC knowledge.

Charged particle correlations at $\sqrt{s}=7 \mathrm{TeV}$ are measured in the typical BEC region. It is observed that an exponential fit to the Fourier space-time transform of the emission source is favoured and that the effective radius of the source reaches a plateau with multiplicity of charged particles. This enhancement of low-Q particle-pair emission is also understood in the context of a three-dimensional QCD string model where the pre- diction of the emission of low mass three-hadron strings at low-Q is observed.

With the introduction of the AFP detectors in ATLAS, the first verification of the selection of a diffractive enriched sample is presented. This result provides good confidence that AFP can provide future measurements of diffractive processes, which offer a bridge between the gap in the soft and hard descriptions of the strong interaction.

\section{References}

[1] ATLAS Collaboration, JINST 3 (2008) S08003.

[2] ATLAS Collaboration, Eur. Phys. J. C71 (2011) 1636.

[3] ATLAS Collaboration, Phys. Rev. Lett. 117 (18) (2016) 182002.

[4] ATLAS Collaboration, Nucl. Phys. B889 (2014) 486-548.

[5] ATLAS Collaboration, Eur. Phys. J. C76 (9) (2016) 502.

[6] ATLAS Collaboration, Eur. Phys. J. C72 (2012) 1926.

[7] ATLAS Collaboration, JHEP 03 (2017) 157

[8] ATLAS Collaboration, Eur. Phys. J. C75 (10) (2015) 466.

[9] ATLAS Collaboration, CERN-EP-2017-092 (in preparation)

[10] ATLAS Collaboration, ATL-PHYS-PUB-2017-012 (2017).

[11] CMS Collaboration, JHEP 09 (2011) 109.

[12] R. Field, Acta Phys. Polon. B36 (2005) 167-178.

[13] Sjöstrand, Torbjörn et al, Comput. Phys. Commun. 191 (2015) $159-177$.

[14] C.-Y. Wong, W.-N. Zhang, J. Liu, P. Ru, Chaoticity and Coherence in Bose-Einstein Condensation and Correlations, in: Proceedings, 10th Workshop on Particle Correlations and Femtoscopy (WPCF 2014): Gyngys, Hungary, August 25-29, 2014, 2015.

[15] I. V. Andreev, M. Plümer, R. M. Weiner, Surprises from boseeinstein correlations, Phys. Rev. Lett. 67 (1991) 3475-3478.

[16] V. A. Schegelsky, A. D. Martin, M. G. Ryskin, V. A. Khoze, Pomeron universality from identical pion correlations at the LHC, Phys. Lett. B703 (2011) 288-291.

[17] M. G. Ryskin, V. A. Schegelsky, Gribov-Regge pomeron and hadron structure phenomenology at high energy, Nucl. Phys. Proc. Suppl. 219-220 (2011) 10-16.

[18] S. Todorova-Nova, Quantization of the QCD string with a helical structure, Phys. Rev. D89 (1).

[19] M. Bahr, et al., Herwig++ Physics and Manual, Eur. Phys. J. C58 (2008) 639-707.

[20] ATLAS Collaboration, CERN-LHCC-2015-009 (2015).

[21] ATLAS Collaboration, JINST 11 (11) (2016) P11013.

[22] G.Anelli, et al., TOTEM Collaboration, JINST 3 (2008) S08007.

[23] ATLAS Collaboration, Phys. Lett. B754 (2016) 214-234. 